Max-Planck-Institut für demografische Forschung

Max Planck Institute for Demographic Research

Konrad-Zuse-Strasse 1 - D-18057 Rostock · GERMANY

Tel +49 (0) 3812081 - 0; Fax +49 (0) 3812081 - 202;

http://www.demogr.mpg.de

MPIDR WORKING PAPER WP 2006-012

MAY 2006

\title{
Forecasts of Cohort Mortality after Age 50
}

Kirill F. Andreev (andreev@un.org)

James W. Vaupel (jwv@demogr.mpg.de)

(C) Copyright is held by the authors.

Working papers of the Max Planck Institute for Demographic Research receive only limited review. Views or opinions expressed in working papers are attributable to the authors and do not necessarily reflect those of the Institute. 


\section{Forecasts of Cohort Mortality after Age 50}

Kirill F. Andreev ${ }^{1}$ and James W. Vaupel ${ }^{2}$

Introductory Note: This report is a revised version of a report completed on June 30, 2004 and prepared for SCOR, a large reinsurance company. The opinions expressed are the authors' and do not necessarily reflect the opinions of SCOR, the United Nations, or the Max Planck Society.

Review of forecasting methods ................................................................................. 1 -

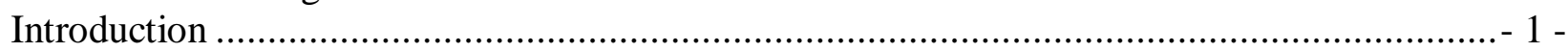

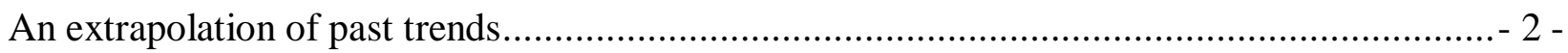

An extrapolation of past trends with explicit assumptions about future mortality levels or rates of

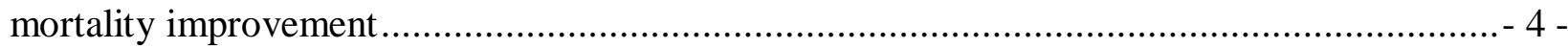

Forecasts by means of model life-tables, reference population, and parameterized functions ... - 5 -

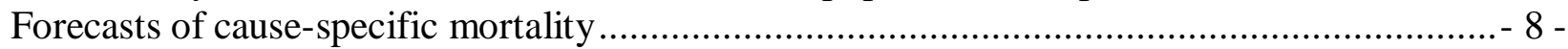

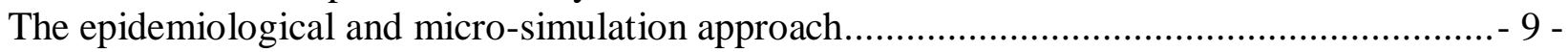

Selected examples of mortality forecasting methodology ................................................ 11 -

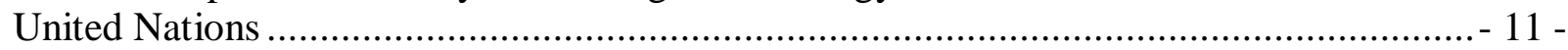

U.S. Social Security Administration .................................................................. 13 -

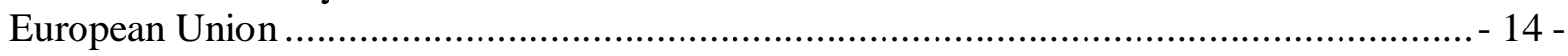

An estimation of mortality levels and past improvement rates ......................................... 15 -

Method 1. The Lee-Carter model with bias correction (LC) . .......................................... 15 -

Method 2. The Lee-Carter model constrained to U.N. forecast of life expectancy (LCEC) ........ - 17 -

Method 3. The Linear Decline model constrained to forecast the Oeppen-Vaupel Line of Life

Expectancy in the Best Practice Country (VAK) ................................................................. 18 -

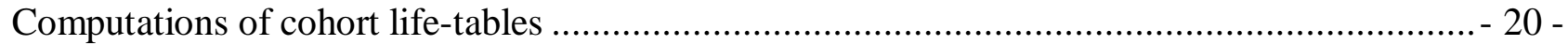

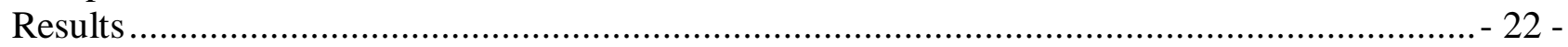

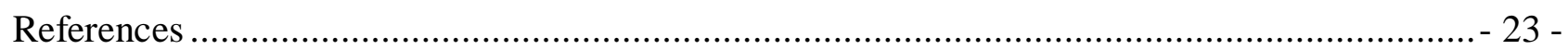

\section{Review of forecasting methods}

\section{Introduction}

Mortality forecasts have several practical uses. First, they are routinely carried out by international organizations (e.g., the United Nations) and by national statistical offices as an integral part of producing population projections. Second, some governmental agencies (e.g, U.S. Social

\footnotetext{
${ }^{1}$ United Nations, Population Division, New York, USA, e-mail: andreev@un.org

${ }^{2}$ Max Planck Institute for Demographic Research, Rostock, Germany, e-mail: jwv@ demogr.mpg.de
} 
Security and Medicare) rely on them when assessing the financial solvency of various governmental programs. Third, pension actuaries employ them in order to determine annuity prices. A large body of literature on mortality forecasting therefore emanates from governmental or actuarial publications rather than from customary scientific journals.

Over the last several decades, a large number of methods for forecasting mortality has been proposed and applied to produce mortality projections. No universal method applicable to all situations has been developed, however. All forecasting methods are based on a few qualitative principles that serve mainly as guidelines for selecting a projection method in a particular situation. All of them, for example, generally agree that the future ahead of us is highly uncertain but that death is an inevitable, though postponeable event. Uncertainty about the future is usually handled by providing high-medium-low scenarios of future mortality development or - a recent tendency — by assigning probabilistic measures to achieve possible outcomes, i.e. stochastic mortalityforecasts. Short-term projections are usually treated separately from long-term forecasts, and the former is based on the general principle that the near future resembles the recent past. Long-term projections are considered to be a more precarious enterprise and the outcome crucially depends on expert opinion as part of the forecasting procedure. All methods in fact rely on expert opinion, though to a varying degree, including those built on seemly pure extrapolative techniques.

Below there follows an attempt to classify forecasting methods, a brief discussion on the advantages and disadvantages of these methods, and relevant references to the literature.

\section{An extrapolation of past trends}

A comprehensive review of extrapolative methods is given by (Pollard 1987). He provides a brief description, discusses the potential strengths and weaknesses of each method, and includes selected examples of their application. According to (Pollard 1987), the simplest method and perhaps one of the most widely used is based on the log-linear extrapolation of observed mortality trends:

$$
\ln _{n} q_{x, t+k}=\ln _{n} q_{x, t}+k \ln g_{x} .
$$

Here, ${ }_{n} q_{x, t}$ is the $n$-ages probability of dying at age $x$ and time $t$, and $g_{x}$ is the annual rate of change in $q_{x}$. An application of this method involves:

a) the selection of "representative" ages (e.g., 0, 1, 5, $10 \ldots 80$ );

b) an estimation of past rates of mortality improvement over some period of time by applying either the endpoint method (e.g., Eq. (1) to compute $g_{x}$ ) or by fitting a regression line (e.g., OLS, WOLS or Poisson); 
c) an application of Eq. (1) to extrapolate the latest available ${ }_{n} q_{x}$ over the forecast horizon;

d) a computation of forecasted mortality rates at intervening ages through an application of interpolated rates of mortality improvement at these ages to the base of the age-specific probabilities of dying.

The procedure is in very close agreement with the description provided by (Pollard 1987). At first glance, extrapolation does not seem to be necessary as historical $q_{x}$ 's are computed by single year of age. The rates of mortality improvement, however, are computed only at the "representative" ages; at the intervening ages the rates are found by interpolation. The reason for using the extra interpolation step instead of directly computed rates of improvement for all ages seems to be dictated entirely by an implicit desire to smooth the age schedule of mortality improvement.

The method hinges almost entirely on the mortality trends observed and to a lesser degree on expert opinion about future mortality. The forecaster, however, has to make several decisions before applying the method. The most important one is on the period to use to estimate mortality improvement rates. This is because the above method explicitly assumes the estimated structure of mortality improvement to prevail for the entire forecast horizon. Additional decisions to be made are to select a) a method for estimating mortality improvement and b) the age aggregation level of the historical data to be used, e.g., five-year age groups ${ }_{5} q_{x}$ vs. single year age groups $q_{x}$. They are less influential as to the final results.

The method is multidimensional despite of its simplicity. It implies that many parameters have to be estimated to make a forecast, but as (Keyfitz 1982) states: "The resulting future mortality estimates can quickly lose their resemblance to the age pattern that we know mortality takes". It is not that unusual that death rates are declining at some ages and increasing at others. This is frequently observed when a short period of time is used for estimating mortality improvement rates, especially when analyzing mortality trends by single-age groups. Projecting such trends in the future will initially distort the age shape of the human mortality curve, a shape that virtually all experts find to be one of the most stable characteristics of the human life span.

Future gains in life expectancy are not known in advance and they are determined both by the estimated age-specific mortality improvement rates and by the death rates in the jump-off year. The relation between the two quantities is not a straightforward one and depends on the life- table entropy ((Keyfitz 1985) (Vaupel 1986).

Over time, many other extrapolative techniques have been published with a view to enhance this simple method. The common variants include 
a) projections of logarithms of central death rates $m_{x}$ rather than $q_{x}$ or logits of $q_{x}$;

b) specifying various constraints on future life expectancy, mortality levels, or on the rates of mortality improvement;

c) constraining future age-specific schedules of death rates to the age patterns dictated by model life-tables or mathematical functions;

d) extrapolating trends in disaggregated population characteristics e.g., by cause of death or by socio-economical status.

Some of these variants are discussed in detail below.

\section{An extrapolation of past trends with explicit assumptions about future mortality levels or rates of mortality improvement}

An assumption commonly used is that $q_{x}$ cannot decline indefinitely; instead the parameter converges to some ultimate level of mortality over time. Eq. (1) is then modified as follows:

$$
{ }_{n} q_{x, t+k}={ }_{n} q_{x, \infty}+{ }_{n} q_{x, t} g_{x}{ }^{k}
$$

where ${ }_{n} q_{x, \infty}$ is the ultimate level of mortality $\left(g_{x}<1\right)$. See (Pollard 1987) for examples and references to an application of this method.

Further variants postulate that mortality drops from some initial level in the jump-off year to some, presumably low, level of mortality over a certain period of time e.g., by n\% over the next several years. Here, the implied rates of mortality improvement generally will be different from the estimated rates of improvement, but the ultimate mortality levels are usually selected in such a way that close agreement between the two series is maintained. Methods similar to those described here have been used extensively by the U.S. Social Security Administration in preparing earlier forecasts of U.S. mortality (Bayo and McKay 1974) (Bayo and Wilkin 1977) (Bayo; Shiman, and Sobus 1978). The forecasts have been produced by extrapolating the death rates for several disease categories. For each cause of death, convergence to some ultimate mortality level has been postulated.

Imposing such constraints on future mortality levels implies that progress made against mortality will eventually stop. The more optimistic view on the development of future mortality is expressed by postulating the ultimate levels of mortality improvement rates rather than the ultimate levels of death rates themselves. If, for example, the rate of improvement is estimated to be $1 \%$ per annum, then one may assume that after a certain time it drops to $0.5 \%$ and continues to remain on this level. Mortality, then, is still projected to decline, and to do so even over the infinite time 
horizon. This approach was adopted, for example, by the Social Security Administration in its most recent forecasts.

In both cases, the selection of ultimate mortality levels or ultimate rates of mortality improvement is based on expert opinion. An expert's view on the future, in turn, is highly influenced by past mortality trends and by errors made in earlier forecasts. The translation of qualitative assumptions about the future into quantitative assumptions of a specific forecasting procedure is usually obscured. Publications provide little information on this process, if any. Sometimes quite a different course of mortality is assumed than that prevailing in the recent period (see, for example, the recent mortality forecasting methodology employed by Social Security Administration and reviewed below).

\section{Forecasts by means of model life tables, reference population, and parameterized functions}

As mentioned above, the distinct form of the age-specific schedule of human mortality is considered by virtually all scholars to be an invariant. No radical changes in the shape of human mortality ever have been postulated. This observation led to the development of model life-tables, relational models, and mathematical functions depicting the age course of human mortality. Constraining future death rates to obey one of the specified forms or to belong to a family of statistical distributions reduces the dimensionality of the forecasting problem and generates more plausible future age-specific schedules of death rates. Such methods (with or without expert opinion on the future levels of life expectancy) are becoming more widely used in forecasting.

The systems of model life-tables (Coale and Demeny 1983;Coale and Guang 1989;Ledermann 1969;United Nations 1982) usually include a collection of age-specific death rates indexed by one or several parameters estimated from the data observed. By selecting an appropriate parameter(s), the entire schedule of age-specific death rates can be generated. The system of model life-tables proposed by (Coale and Demeny 1983) involves only two parameters, for example. First, a family of life tables has to be selected, e.g., "West". Second, the future levels of life expectancy need to be chosen either by extrapolation or by relying on expert opinion or by a combination of both. Given the level of life expectancy for each year, the age-specific schedule of death rates is selected from the model life-table system, an approach that is used extensively by the (United Nations 2000a), (United Nations 2001b) and in several European countries (Cruijsen and Eding 2001).

The main advantage of such method is that the projected age-specific schedules of death rates are in agreement with the age-specific patterns observed earlier. A disadvantage is the 
arbitrariness involved in the selection of future life-expectancy levels, thus imposing a very strong constraint on future mortality levels. Additionally, producing a forecast for low mortality populations may be problematic as the high levels of life expectancy anticipated for the future may be outside the range of the life expectancy levels covered by the model system (Coale and Guang 1989; Buettner 2002). A review of the methods employed by the United Nations (see below) provides additional information.

A method closely related to that of model life-tables is to make forecasts by reference to a more advanced population. This method assumes that a population for which a mortality forecast has to be produced follows the mortality history of a more advanced population. The latter is chosen beforehand and the time lag between both populations is specified. By assuming, for example, that the time lag is invariant over time, the future death rates are simply equal to those observed in the more advanced population. A common variant includes the postulation of future life-expectancy levels instead of the time lag. Age-specific schedules of death rates are then selected from the more advanced population, given a life expectancy level. In this case, the more advanced population serves as a model life-table system for the current population.

This method has been one of the mainstream methods of mortality projections and several examples of its application are provided by (Pollard 1987). The advantages and disadvantages of the method are comparable with the methods based on model life-tables. Recent accumulations of mortality data in publicly accessible databases (Human Mortality Database 2004) provide more empirical material for further applications of this method. Moreover, the assumption of a constant lag between advanced populations and those catching-up can be further relaxed as there is substantial evidence that some countries catch up faster or lag significantly behind, thus reducing or increasing the time lag (Wilmoth 1998;Oeppen and Vaupel 2002).

Another class of mortality forecasting methods is based on relational mortality models. Producing a forecast involves fitting a relational model to the mortality trends observed and forecasting the time series of the parameters of a selected model. The constraints on future life expectancy can be specified, too. Most of the methods are rooted in the Brass relational model and its extensions (Brass 1971;Lopez; Salomon; Ahmad; Murray, and Mafat 2000;Zaba 1979;Zaba 1989;Ewbank; Gomez De Leon, and Stoto 1983;Hannerz 2001;Murray; Ferguson; Lopez; Guillot; Salomon, and Ahmad 2003).

The Brass two-parameter logit model system is based on a linear relation between the logit of the survival function in the current population and the logit of the survival function in a standard or reference population: 


$$
\Lambda_{x}=\alpha+\beta \Lambda_{x}^{s}
$$

where $\Lambda_{x}=\frac{1}{2} \ln \left(\frac{l_{0}-l_{x}}{l_{x}}\right)$. If $\Lambda_{x}^{s}$ is defined by a period life-table at the beginning of the fitting period, then by fitting (3) to $\Lambda_{x}$ in each calendar year time-series $\alpha(t)$ and $\beta(t)$ can be estimated. These series of parameters can be used further to produce a forecast by means of time-series methods. The future age-specific schedules of death rates then are generated in compliance with the Brass model.

A straightforward extension of this method involves employing a more complicated relation model (e.g., see (Zaba 1979)) usually providing a closer fit to the observed data. This inevitably leads to increasing the dimensionality of the problem, thus making the forecast more difficult. For recent experiments with the Brass logit model, see (Lopez and others 2000).

Further, instead of employing the Brass model the observed death rates can be fitted by a mortality model or by a mortality "law". The estimated time series of the parameters can be extrapolated into the future, thus producing a mortality forecast. This approach was adopted by (McNown and Rogers 1989), who fitted the Helligman-Pollard Mortality model (Heligman and Pollard 1980) to the annual series of age-specific death rates in the United States and then extrapolated the fitted parameters by means of standard time-series methods. The time series of the parameters exhibited highly non-stationary behavior and this made it difficult to select a proper model for extrapolation.

The dimensionality of the forecasting problem also can be reduced by looking at mortality at adult or older ages. Mortality models proposed to depict death rates at adult and advanced ages ((Thatcher; Kannisto, and Vaupel 1998)) include fewer numbers of parameters than do those constructed for the entire life span. Forecasting the time series of parameters is expected to be easier in this case. We did not find, however, any publications based on this approach. (Bongaarts 2004) recently suggested use of Kannisto's model for long term projections but his article did not provide other important details on forecasting, such as a method for forecasting life expectancy.

Another class of extrapolative methods closely related to the procedures discussed here includes so-called targeting methods. For forecasting purposes, a target age-schedule of death rates is selected (e.g., from another, more advanced population) and convergence to this schedule is postulated over a certain period of time. See Preston (1974) for an application of such methods.

Generally, the methods described in this section are geared to short-term rather than longterm forecasts. Also, they are more suited to developing than to developed countries. The methods based on model life-tables or the reference to a more advanced population do not allow 
extrapolation beyond the observed levels of life expectancy. It is still possible, however, to do so by forecasting the time series of the mortality model parameters but the age shape of the death rates is not necessarily preserved unless forecasting is done within a restricted parameter space, e.g., by postulating a certain correlation between the parameters or by specifying a certain level of life expectancy in the future. The latter is especially difficult as expert opinion regarding future levels of life expectancy differs widely due to the lack of a well established theory of human longevity.

\section{Forecasts of cause-specific mortality}

Trends in total mortality can be disaggregated by cause of death, and future death rates can be obtained by forecasting death rates for each cause of death separately. The subsequent aggregation of the resultant trends produces a forecast of total mortality. This method has an undisputable advantage in that the examination of cause-specific mortality provides additional insights into the determinants of mortality trends. This may be important when it comes to producing short-term forecasts. An example provided by (Pollard 1987) shows that the method was successful in predicting the leveling-off of Australian male mortality in the 1960s. All other methods failed to predict this attenuation of the mortality trend. Death rates in pre-1960s Australia rapidly declined due to reductions in infectious mortality whereas mortality from cardiovascular diseases and to a lesser extent from accidents climbed. A failure to account for these structural shifts in overall mortality by other methods resulted in the failure to predict the leveling-off of death rates in the 1960s. A similar observation was made by (Preston 1974) on Canadian mortality projections for the 1950s. The importance of incorporating cause-specific mortality statistics in the mortality projections is further stressed by (Caselli 1996).

Other authors argue that the use of causes of death does not significantly improve the accuracy of mortality forecasts (Alho 1991) and that great care should be taken in forecasting causes of death that are rapidly changing (McNown and Rogers 1992). Also, the simple extrapolation of cause-specific death rates apparently tends to produce more pessimistic forecasts of total mortality since the causes of death that were stable or increasing over the fitting period will eventually determine the dominant trend in overall mortality (Wilmoth 1995).

Demographic methods commonly practiced rely on the assumption that different causes of death are independent of each other: The observed trends in one cause of death are forecasted without taking into account trends in other causes of death. Trends in different causes of deaths are correlated, however. The reduction or elimination of one cause of death is related to changes in specific risk factors prevailing in the population, the emergence of new forms of medical treatment, 
and public health measures. These factors, in turn, may affect several causes of death simultaneously, thus generating a dependence between cause-specific mortality trends. The great reductions in deaths from infectious diseases over the $20^{\text {th }}$ century and the virtual eradication of several mortal diseases triggered by the discoveries of Louis Pasteur and Robert Koch is a prominent example. Generally, we cannot predict the effect of falling death rates from one cause of death on another without making additional assumptions (Yashin, Manton, and Stallard, 1986). The problem of competing risks is frequently discussed in this context. It is still unresolved and so far no adequate solution has been found, and this despite its great importance to the development of mortality projections.

Another difficulty with this approach is related to the estimation of death rates by cause of death. The relevant statistics generally are of inferior quality compared to those on total mortality. The reconstruction of consistent trends by cause of death is a challenging task owing to several revisions to ICD classification, changes in coding practices, improved diagnostics, and multiple conditions of death at older ages. Generally declining rates of autopsies worldwide introduce additional uncertainty ((Loughrey; McCluggage, and Toner 2000;Chariot; Witt; Pautot; Porcher; Thomas; Zafrani, and Lemaire 2000;Lindstrom; Janzon, and Sternby 1997).

In sum, an examination of the recent trends in cause-specific mortality appears to be important in producing short-term forecasts. Projections by cause of death may provide an adequate description of the future mortality course over a short period of time. Cause-specific forecasts are of less benefit to long-term forecasts than they are to the short-term variant, however, due to the current lack of knowledge about disease etiology and about the factors underlying mortality trends in the distant future. The Dutch Statistical Office, for example, examines cause-ofdeath specific statistics for the interpretation of recent mortality trends and for making qualitative assumptions about future mortality development. The mortality projections themselves are produced for total mortality without disaggregation by cause of death (Van Hoorn and De Beer 2001).

\section{The epidemiological and micro-simulation approach}

Following this approach, mortality is generally explained by risk factors, such as smoking, obesity, the socio-economic status, environmental exposure, and the genetic endowment prevailing in the population (see e.g., (Van Den Berg Jeths; Hoogenveen; De Hollander, and Tabeau 2001)). The mortality projection can be carried out by extrapolating the risk factors into the future. The 
extrapolation itself usually takes the form of different scenarios, resulting in different mortality forecasts.

The projection of mortality arising from lung cancer carried out by (Alderson and Ashwood 1985) serves an illustration of this approach. The authors developed a regression model, utilizing available historical data to describe the relationship between the lung-cancer death rate and smoking patterns, and applied the model to the population of England and Wales. The model was fitted to historical data and the future death rates of lung cancer mortality predicted by extrapolating the current smoking patterns into the future, using two different scenarios (Van Den Berg Jeths and others 2001).

Further advancement in this field led to the development of models simulating demographic and epidemiological processes of diseases and mortality at individual levels, namely dynamic multistate models and stochastic microsimulation models. A comprehensive microsimulation model was developed by Statistics Canada (Wolfson 1994) for the health monitoring of the population of Canada (POHEM-population health model). By using information on many demographic, socioeconomic, and risk factor variables, the model simulates the future dynamics of the Canadian population, using several models describing demographic and epidemiological processes. The future health status of the Canadian population as to mortality then can be calculated from the simulated microdata.

A similar approach was used by (Manton; Stallard, and Tolley 1991) to assess the theoretical limits to life expectancy by means of a risk-factor model (Woodbury and Manton 1977). An individual's chances of death were assumed to be dependent on the level of "senescent" mortality captured by the Gompertz distribution and to depend on a combination of individual risk factors. The model was applied for the estimation of life expectancy attainable under optimal trends in the risk factor variables.

Following this approach, a mortality forecast can be produced by estimating the trends in the distribution of the risk factors in the population and by extrapolation them into the future. An application of this model, however, is hampered by the lack of adequate individual data. The necessary data on individual risk factors are available from longitudinal survey data (e.g., the Framingham Heart Study or the National Long Term Care Survey). These, however, cover a tiny share of the total population only. The applicability of estimates obtained from such data to predict the mortality of the total population therefore is questionable.

In sum, risk-factor approaches may prove useful in developing the methodology of mortality forecasting. The relation between risk factors and mortality, however, is imprecise and future trends 
in risk factors are uncertain (although to some degree they are controllable), thus presently limiting the application of such models.

\section{Selected examples of mortality forecasting methodology}

\section{United Nations}

Starting in 1951, the United Nations Population Division has been producing population estimates and projections for all countries in the world. Several assumptions about future fertility, mortality, and migration have been made and several variants of population projections published (United Nations 2001a) (United Nations 2003). Three mortality assumptions have been specified: normal, no-AIDS, and constant mortality. The normal assumption projects the future level of life expectancy for each country on the basis of models that capture changes in life expectancy, models produced by the United Nations Population Division. For countries severely affected by HIV/AIDS epidemics, estimates of its impact have been made by projecting the yearly incidence of HIV infection. As the future levels of life expectancy are determined, the age-specific death rates are calculated for the projection period by means of either model life-tables or by a combination of model life-tables and observed age-specific mortality. A decision as to which method to use is made by the analyst and this decision is influenced by the data available for a particular country (United Nations 2001b;Buettner 2002).

The main step in producing the forecast therefore is to figure out future life expectancy. An appropriate model is chosen for any given country "by taking into account the observed pace of mortality decline in the recent to medium-term past". A closer look reveals that the appropriate model is just a set of pre-defined paces of increase in life expectancy over time (Table VI.13, (United Nations 2001b). There are three different scenarios for males and females: a fast, medium, and slow pace. It is also generally assumed that the higher the level of life expectancy is, the slower is the pace of any likely future increase. For the fast pace variant (for example, the level of female life expectancy lies in the range of 55.0-57.5 years), the pace of increase is five years per decade; if it is between 80.0 and 82.5 years, the pace is permitted to be a mere 1.6 years per decade. After 2025, life expectancy is assumed to increase according to the medium-paced model regardless of the model selected for earlier years. No justification other than to ensure "international consistency" (Buettner 2002) is provided, i.e., information on how such models have been developed and how the analysis of past trends leads to the selection of a model for life expectancy levels in the future is missing. 
The next step in constructing United Nations mortality forecasts involves the selection of age-specific death rates for the forecast horizon. Details of this process are obscure. As the ultimate goal was to produce population estimates, the main concern perhaps was to calculate the survivor ratios needed for advancing the current population by five years. This was done by computing the survivor ratios implied by the current life table, assuming that the death rates in the life table prevail over the entire five-year period. The model life-tables therefore served "to establish a plausible and consistent pattern of change for survivor ratios as the expectation of life at birth changed, but it did not determine the pattern of mortality. Irrespective of the underlying model life-table chosen, the mortality pattern converged to a life-table characterized by an expectation of life at birth of 82.5 years for males and 87.5 years for females".

The need for producing long-term forecasts for high longevity countries led the United Nations Population Division to revise their methodology (Buettner 2002). First, after having realized just how important mortality developments are at very old ages, the Population Division extended the range of model life-tables from ages $80+$ to ages $100+$. A relational model developed by (Himes; Preston, and Condran 1994) was employed for this purpose. Second, the Division extrapolated the existing model life-tables into the higher levels of life expectancy, using the LeeCarter model (Lee and Carter 1992), specifically adopted to perform this task. As the model lifetables depend on a single index, i.e. life expectancy at birth, they were ranked in terms of life expectancy rather than calendar time, and the Lee-Carter model was fitted to such series of life tables, with the $k_{t}$ parameter modified to be parameter $k_{l}$, reflecting the current level of life expectancy instead of mortality conditions in the current calendar year (as in the original model). Even if the series of life tables produced by the extrapolation of $k_{l}$ passed visual inspection, the observed trends in $k_{l}$ no longer were linear, and several additional steps have been made to produce model life-tables for higher levels of life expectancy (Buettner 2002).

One of the recent undertakings of the United Nations Population Division has been to produce forecasts of national populations up to 2300 (United Nations 2004) - the longest forecast ever attempted. Mortality for the 2050-2300 period has been forecasted by the Lee-Carter model fitted to the death rates projected for the years 2000-2050 and produced earlier. The model was fitted to both sexes combined and then applied separately to each sex in order to achieve reasonable sex differentials in mortality. 


\section{U.S. Social Security Administration}

Mortality projections prepared by the U.S. Social Security Administration forms part of an actuarial analysis aimed at assessing the projected financial solvency of the Social Security program. The latter is financed through two separate trust funds: (a) the Old-Age and Survivors Insurance (OASI), and (b) the Trust Fund, and The Disability Insurance (DI) Trust Fund (collectively known as OASDI). The OASI fund provides monthly benefits to retired workers, their families, and to survivors of deceased workers. The DI fund pays disabled workers and their families monthly benefits. Each year the Board of Trustees releases a report on the financial solvency of the Social Security program, among other factors based on several mortality assumptions. The demographic assumptions underlying the report are used to estimate the future size of the beneficiary population. The mortality forecast aims at a horizon of 75-years and is made by the Office of the Chief Actuary (OCACT), which forms part of the SSA.

The methodology of mortality forecast is described in actuarial publications of the SSA (Bell; Wade, and Goss 1992) (Bell and Wade 2002). Every four years the Social Security Advisory Board (SSAB) appoints the Technical Panel on Assumptions and Methods. The latter's task is to review the assumptions underlying the Trustee's report (2003 Technical Panel on Assumptions and Methods 2003).

The SSA mortality forecasts are based on an analysis of historical trends in death rates by seven disease categories: heart disease, cancer, vascular disease, violence, respiratory disease, diabetes mellitus, and a residual group of diseases (Bell and Wade 2002). The rates of mortality decline by sex and the causes of deaths are estimated for the 1979-1999 period as well as for future rates of mortality improvement. The estimated rates of decline are assumed to prevail over the next two years and then to converge to the postulated ultimate rates of decline. The convergence is assumed to take place by 2026 and the ultimate rates to apply after this year. When death rates were found to increase over the years 1979-1999, the decline was nevertheless postulated over the long run. For example, for age group 85 and over it was only the death rates arising from heart and vascular diseases that fell; the rates for all other causes of death increased, as did total mortality. Nonetheless, the ultimate rates of mortality improvement were assumed to be positive for all of the disease categories. The SSA publications do not contain information on the qualitative assumptions affecting these decisions and on their translation into quantitative assumptions underlying the forecasting method.

A comprehensive review of successive forecasts produced by the SSA has been conducted by (Olshansky 1988). One of the observations made was that the forecasts were generally 
pessimistic. A recent change in methodology from postulating the ultimate level of death rates to postulating the ultimate rates of decline reflects the tendency to produce more optimistic forecasts. Postulating the ultimate levels of death rates implies that no further progress is made in reducing the mortality rate once the ultimate levels are reached. By postulating ultimate rates of decline, death rates are assumed to be falling over the entire forecast period. The methodology used for producing earlier forecasts can be found in publications of (Bayo and McKay 1974), (Bayo and Wilkin 1977), (Bayo and others 1978) and (Bell and others 1992).

\section{European Union}

The assumptions, results, and methodology of the mortality forecasts carried out by countries of the European Union over the 1992-1997 period have been reviewed by (Cruijsen and Eding 2001). The authors generally conclude that the methods used in forecasting mortality varied broadly between the countries: from purely extrapolative techniques (Belgium, France, Luxembourg, and Italy) to specifying targets attained at the end of the forecast horizon (Austria, Germany, and Sweden) or a combination of both (Denmark, Finland, Ireland, Spain, the Netherlands, and the United Kingdom).

Large variations also existed within the groups. For example, whereas the forecast for Belgium was produced by extrapolating observed age-specific mortality trends using a mathematical function, the forecast for Italy was based on quantitative assumptions about causespecific mortality. An age-period-cohort model was applied to the regional Italian data in order to produce a forecast of total mortality.

As to the targeting methods, the targets varied from life tables borrowed from a more advanced population (Germany) to model life-tables developed by Coale and Guo (Austria), or a combination of a model and national life tables (Sweden). Some countries used a combination of extrapolation and targeting. Forecasts for the Netherlands, for example, were produced by analyzing recent trends in cause-specific mortality, extrapolating life expectancy for a short period by means of a spline function and by specifying a life expectancy target at the end of the forecast horizon chosen according to expert recommendations. The forecast itself was carried out only for total mortality and not by cause of death (Van Hoorn and De Beer 2001).

(Cruijsen and Eding 2001) observed that most of the mortality forecasts became more optimistic when compared with those made around 1985. All countries foresee further increases in life expectancy but generally at a lower pace than that observed in the previous two decades. 


\section{An estimation of mortality levels and past improvement rates}

The mortality data for this project stems from the (Human Mortality Database 2004) (HMD), which includes life tables by single year of age and by single calendar year for many developed countries. The HMD also contains the methodology used for computing life tables, underlying raw population and vital statistics data and references to the original data sources, making the link between the collected data and the final mortality estimates transparent for the user. Using a common methodology for all countries ensures that the observed differences in survival are due to underlying differences in the data on population and deaths rather than to different approaches adopted by national statistical offices in preparing mortality estimates.

For this project, the latest data was used whenever possible. The data may not yet be available on the public web site of the HMD. The rates of mortality improvements have been estimated according to the equations provided in the original consultancy agreement.

\section{Method 1. The Lee-Carter model with bias correction (LC)}

The Lee-Carter model (LC) is an extrapolative type model that is not based on population theory or expert opinion (Lee and Carter 1992). It stipulates that logs of death rates over age $x$ and time $t$ are well approximated by the following formula:

$$
\ln \mu_{x t}=a_{x}+b_{x} k_{t}+\varepsilon_{x, t}
$$

where $a_{x}$ is an average age effect, $k_{t}$ is "an index of general mortality level" and $b_{x}$ shows how sensitive the death rate at age $x$ is to changes in $k_{t}$. For complete identification of the model, the following constraints are usually imposed: $\sum_{x} b_{x}=1$ and $\sum_{t} k_{t}=0^{3}$. The model approximates the observed matrix of death rates. It can be applied to any age range and age group. For example, we can fit it to death rates available by single year of age (e.g., 0, $1 \ldots 100+$ ) and by single calendar year (e.g., 1950, 1960 ... 2000). Alternatively, we can choose to fit it to adult ages only (e.g., for ages 50 and over). In practice, the model has been applied and rigorously tested on the data aggregated in 5-year age groups with the following open age groups: $85+90+$ or $100+$. As it is not clear what effect the data aggregation has on mortality forecasts, we apply the model to the data aggregated into 5-year age groups even if the original data are available by single year of age.

\footnotetext{
${ }^{3}$ If $\sum_{t} k_{t}=0, a_{x}$ is simply an average of $\ln \mu_{x, t}$ over time.
} 
The model has been estimated by a method originally suggested by (Lee and Carter 1992). First, estimates of $a_{x}$ are computed by averaging $\log m_{x, t}$ over time. Second, $b_{x}$ and $k_{t}$ are estimated by singular value decomposition applied to the adjusted matrix of death rates $\log m_{x}-a_{x}$. Finally, the $k_{t}$ vector is adjusted in such a way that the observed total number of deaths in the current year is equal to the number of deaths predicted by the model.

The mortality projection is carried out by forecasting time series of $k_{t}$ by a random walk with the drift model (RWD) (also known as the "ruler" method of forecast as it connects the first and last points of available data with a ruler and then extends the resulting line further to produce a forecast) assuming that $a_{x}$ and $b_{x}$ are constant over time:

$$
k_{t}=k_{t-1}+c+\varepsilon(t) .
$$

The forecast starts with death rates observed rather than fitted by the model in the last year with available data. This is different from the original procedure and is known as "bias-correction"; see (Bell 1997), (Lee and Miller 2001) and (Lee 2000) for further details. This helps to eliminate a jump between observed and forecasted death rates in the first year of the forecast as the model does not fit age-specific death rates exactly in the last year. If the fitting period is sufficiently long, then the difference between the observed and the fitted death rates can be appreciable.

Using the RWD model for forecasting $k_{t}$ is equivalent to forecasting each age-specific death rate to decline at its own rate ((McNown 1992). Indeed, as it follows from (5), $\ln m_{x, t+1}-\ln m_{x, t}=b_{x}\left(k_{t+1}-k_{t}\right)=b_{x} c$ : The ratio of death rates in two subsequent years of the forecast is equal to $b_{x} \cdot c$ and invariant over time. Product $b_{x} \cdot c$ therefore is equal to the rate of mortality change over time at age $x$ and parameter $b_{x}$ can be interpreted as a normalized schedule of agespecific rates of mortality change over time.

The method relies on one assumption only, namely that $b_{x}$ is held in the future and that all changes in the course of mortality are captured by the $k_{t}$ parameter. All other statistics of future survival distributions (e.g., life expectancy) are computed from the forecasted death rates. This method does not impose specific constraints on future life expectancy levels or on the maximum life span; their forecasted values depend only on the estimated parameters and on the method for forecasting $k_{t}$. In practice, though, no methods other than the random walk with drift has been applied to the extrapolation of this parameter (Booth; Maindonald, and Smith 2002).

Originally, the method was proposed for producing stochastic mortality forecasts, with the only output being a confidence interval of future life expectancy levels. If it is applied to produce a 
point interval, then it will yield almost the same result as by a simple extrapolation of $\log m_{x}$ (see also Equ. 1). Note that the method was not intended for producing long-term forecasts.

The SCOR Method 1 is an application of this procedure, fitted to data for age groups 50-54, 55-59 ... 100+ and for the years from 1950 to the latest year available.

\section{Method 2. The Lee-Carter model with External Constraint (LCEC), constrained to the U.N. forecast of life expectancy}

The LC method was originally proposed to produce short and medium term forecasts. If it is used as a point forecast for long term projections, then its performance may significantly deteriorate. Several factors largely influencing the performance of the LC method are noted by McNown (1992), Lee and Miller (2001), Alho (2000), Booth et al. (2002), Alho (2000). One way of gaining more stability over the long run is to reduce the freedom of forecasts by imposing a constraint. For example, we can constrain the forecast to be in agreement with the period life expectancy computed by a separate procedure, either of our own or published by some agency. Then we can combine the forecast of life expectancy with the LC forecast in order to obtain a complete set of age-specific death rates.

We develop this idea as follows. First, we fit the LC model (4) to the data by single year of age $0,1 \ldots 100+$ and by single calendar year, starting from 1950 and ending at the latest year available. The forecast of death rates for the next year is computed by multiplying vector $b_{x}$ by the annual change in the $k_{t}$ parameter:

$$
\Delta \ln m_{x, t}=b_{x} \Delta k_{t},
$$

where $\Delta \ln m_{x, t}=\ln m_{x, t+1}-\ln m_{x, t}$ and $\Delta k_{t}=k_{t+1}-k_{t}$.

In the original LC method, $\Delta k_{t}$ is constant over time and it is equal to the estimates of a drift parameter of the RWD model fitted to the series of $k_{t}$. In the method proposed here, $\Delta k_{t}$ is computed for each year in such a way that

$$
e_{0, t+1}^{*}=e_{0, t+1}\left(\Delta k_{t}\right),
$$

where $e_{0, t+1}^{*}$ is an external constraint on life expectancy at birth and $e_{0, t+1}\left(\Delta k_{t}\right)$ is the forecasted life expectancy that depends on $\Delta k_{t}$. Parameter $\Delta k_{t}$ is found numerically by computing a root of the function returning the difference between $e_{0}^{*}$ and $e_{0}$. 
In general, $\Delta k_{t}$ will not be constant over time; instead it will follow a curve capturing the difference between $e_{0}^{*}$ and $e_{0}$. Parameter $b_{x}$ (the normalized age-specific pattern of mortality change over time) is still constant and does not depend on time. This implies that each age-specific death rate no longer is forecasted to change at its own rate, rather the rate will vary pending on the life expectancy constraint.

SCOR Method 2 is a variant of this method, with the life expectancy constraint taken from the U.N. projections (United Nations 2000b). As U.N. life-expectancy forecasts are available by quinquennial periods only, life expectancy by single calendar year has been obtained by linear interpolation. For years beyond the U.N. forecast horizon, life expectancy is assumed to be constant at the level of the latest value available. More information on how the U.N. forecast of life expectancy is produced is provided elsewhere in this report and in the respective documents of the United Nations.

To produce forecast for ages 50 and over, no additional adaptation of this method is required except that the constraint on life expectancy at birth must be replaced by the constraint on life expectancy at age 50. Because the U.N. projections of life expectancy are available only for life expectancy at birth, we used this method for the entire age range rather than for ages 50 and above.

\section{Method 3. The Linear Decline model constrained to forecast the Oeppen-Vaupel Line of Life Expectancy in the Best Practice Country (VAK-Vaupel, Andreev, Kannisto)}

This is a new method of forecast and makes use of an observation on the trend in life expectancy in the best practice country (Oeppen and Vaupel 2002). Assume that the linear trend in best practice female life expectancy continues into the future. Assume also that the difference between the life expectancy of a particular country and the general trend stays constant over time. Then we can forecast life expectancy at birth as:

$$
e_{0}(y)=e_{0}\left(y_{\text {last }}\right)+s\left(y-y_{\text {last }}\right),
$$

where $s$ is the pace of increase in best practice life expectancy over time. Parameter $s$ has been estimated by (Oeppen and Vaupel 2002) to be equal to 0.243 for females and 0.222 for males. Taking into account the convergence of mortality differentials between the sexes observed over the last decade in several developed countries, we do not choose separate values of $s$ for males and females but rather use an estimate of $s$ for females throughout. 
In view of forecasting life expectancy at birth, we need to estimate life expectancy at age 50 . To do so, we start with a forecast of death rates by the Linear Decline model ${ }^{4}$, very similar to (1). Then we multiply the forecasted death rates $m_{x}$ by a constant term $c$ chosen in such a way that life expectancy at birth is equal to the life expectancy implied by Eq. 8. Finally, $e_{50}$ is extracted for further computations from the period life-tables calculated from the adjusted death rates. Note that it is not necessary to forecast death rates at all ages; only those below age 50 are required for calculating $e_{50}$.

Given $e_{50}$, we need to compute the age-specific schedule of death rates that correspond to this level of life expectancy. We use the Kannisto model of mortality for this purpose as (a) it appears to be very simple, complying with requirements specified by (Keyfitz 1982), and (b) it is still flexible enough to be applicable over a long period of time (Thatcher 1999):

$$
\mu(x)=\frac{a e^{b x}}{1+a e^{b x}} .
$$

How can we determine parameters $a$ and $b$ corresponding to $e_{50}$ ? After extensively investigating the fit of this model to historical data, we adopt the following procedure. First, we fit the model ${ }^{5}$ to the annual period life tables in order to estimate the trends in parameters $a$ and $b$. Then we fit an ordinary linear regression to determine the rate of decline in $\ln a$ over time given by $\beta_{1}$ :

$$
\ln a=\beta_{0}+\beta_{1} t .
$$

We use recent years only to fit this regression model, and the length of series for fitting the regression visually was found to provide a good fit $\left(R^{2}>=0.97\right)$. By assuming that the decline in $\ln a$ will continue over the entire forecast horizon, parameter $b$ can be found from the current $\ln a$ and the $e_{50}$ values. For the first year of forecast, this method may generate a jump in death rates. Whether or not this jump occurs depends on how well death rates above age 50 are approximated by the Kannisto model ${ }^{6}$. To ensure smooth transition from the observed death rates to the death rates implied by this model, the forecasted death rates were blended with the death rates produced by the LC procedure over a short period of time.

The LC death rates were produced by applying this method to the data for ages $50,51 \ldots$ $100+$ constrained to life expectancy at age $50, e_{50}$. The LC forecast fires off with death rates

\footnotetext{
${ }^{4}$ According to this model, each age-specific death rate is forecasted to decline at its own independent rate. We use the RWD models fitted separately to the log of death rates in each age group.

${ }^{5}$ This model was fitted to data for ages 50 and over by the MLE method maximizing likelihood implied by the Poisson distribution of deaths. The first age, 50, was subtracted from the AGE variable before including it in the likelihood, mostly for the sake of numerical stability.

${ }^{6}$ For example, the Kannisto Model fits data extremely well for England and Wales, Males, 1997, but it lacks fit for ages below 60 for France, females, 1997. In the first case, the jump will be hardly noticed whereas in the second case the transition from the observed to the forecasted death rates will be quite abrupt.
} 
observed in the last year producing death rates in the first years of forecast to be quite similar to those observed in the most recent years. Therefore, we assign more weight to the LC death rates at the beginning of the blending period whereas the death rates returned by the Kannisto model receive more weight at the end of this period.

Let $n$ be the length of the blending period (it always starts with the first year of forecast) and $w$ be the blending weights. We use linear weights independent of age:

$$
w_{i}=\frac{i}{n+1} ; \quad i=1 . . n .
$$

For all ages from 0 to 100 and for any year inside the blending period, the resulting death rate $m$ is given by a weighted sum of death rates,

$$
m_{V A K}=w_{i} m_{\text {KANNISTO }}+\left(1-w_{i}\right) m_{L C} .
$$

For the years beyond the blending period,

$$
m_{V A K}=m_{\text {KANNISTO }} .
$$

We use $n=10$ for populations where the Kannisto model provides a good fit to the observed data and $n=40$ where it does not do so.

The LC forecasts are available only up to age 100+, so starting with age 101 we are not able to blend the death rates produced by the two methods. This possibly introduces another jump in death rates at age 100. To prevent this jump, a weighted least square line was fitted to the death rates at ages 98, 99 .. 103 with the U-shaped weights (4 211124 ). The death rates at ages 99, 100, 101, and 102 were replaced subsequently with the predictions of this model.

All of these adjustments to the death rates possibly lead to a slight deviation in the resulting life expectancy at age 50 from the original life expectancy trajectory based on the Oeppen-Vaupel Line. The deviation is not expected to be large but as final adjustment we will nevertheless multiply the death rates in each year of the blending period by an adjustment factor chosen in such a way that the resulting life expectancy will be exactly equal to the original.

\section{Computations of cohort life-tables}

Cohort life tables have been produced from the forecasted period death rates by computing the age-specific probabilities of dying, using Lexis triangles. All forecasting methods produce a matrix of death rates by calendar year and age groups. If, for example, a method produced a death rate for age group 50-54 and for the year 2050, we assume that the death rates in all of the ten Lexis triangles constituting this year-age element are equal to the forecasted death rate. 
The death rates in open age intervals are not split by Lexis triangles; rather we assume that mortality is constant. The VAK method has no open age interval but technically computations have been carried out only up to age 120 , assuming that ${ }_{\infty} m_{120}$ is equal to the $m_{120}$ returned by the Kannisto model.

As a result, we have two death rates for any cohort $z$ and age $x: m_{L}$ is a death rate in the lower Lexis triangle and $m_{U}$ is a death rate in the upper one, each of them refers to a half-year ageinterval (note, that $m_{L}$ pertains to year $x-z$ and $m_{U}$ to the next year). Assuming that the average time lived by persons who died is 0.25 years in a Lexis triangle, we can convert the two death rates into probabilities of dying in each of the Lexis triangles (sometimes called "partial probabilities of dying”), using the standard demographic equation (Preston; Heuveline, and Guillot 2000):

$$
{ }_{n} q_{x}=\frac{n \cdot{ }_{n} m_{x}}{1+\left(n-{ }_{n} a_{x}\right)_{n} m_{x}}
$$

with $n=0.5$ and ${ }_{n} a_{x}=0.25$. Parameter $q_{x}$ by single year of age is then computed as $q=1-\left(1-q_{U}\right)\left(1-q_{L}\right)$. Finally, a cohort life table is constructed by standard methods, with $a_{x}=0.5$ for all ages.

These cohort life tables are based on raw forecasted death rates. The age-specific pattern may be irregular because of random fluctuations and because of splitting 5-year death rates into Lexis triangles, as is the case with the LC model. The second set of life tables is aimed at addressing this issue by smoothing the raw death rates before computations of the rest of the life table can be done. The smoothing is carried out by a cubic spline $f\left(x_{i}\right)$. Spline $f$ is found by minimizing the following objective function (de Boor 2001):

$$
p \sum_{i} w_{i}\left(\ln m_{i}-f\left(x_{i}\right)\right)^{2}+(1-p) \int f^{\prime \prime}(x)^{2} d x,
$$

where $\ln m_{i}$ are logs of raw death rates, $w_{i}$ are weights and $p$ is a parameter governing the smoothness of the resulting spline $f$. Usually, weights reflect the reliability of estimated death rates $w_{i} \propto \frac{1}{\operatorname{Var}\left(m_{i}\right)}$ but because cohort life tables include both observed and forecasted death rates, we use $w_{i}=1$ throughout.

Parameter $p$ varies between 0 and 1 governs the smoothness of the resulting spline. For $p=$ 0 , the spline is simply a least-square straight line fit to the $\ln m_{i}$. For $p=1$, it is the "natural" cubic spline interpolant passing exactly via each $\ln m_{i}$. Choosing a specific value of $p$ for our purposes is not obvious and we tried several standard algorithms for the automatic selection of $p$. 
No method was found to be adequate for all purposes. If, for example, $p$ is chosen by minimizing the cross-validation statistics, then smoothing for the LCEC and VAK methods is acceptable but for the LC method the resultant spline follows the five-age steps of the forecasted death rates too closely.

Therefore, instead of looking for a parameter value of $p$, we decided to specify the smoothness of the spline in advance. Let $\Delta^{2} \ln m_{x}=\ln m_{x+2}-2 \ln m_{x+1}+\ln m_{x}$ be the second order difference $^{7}$ and $\gamma=\frac{1}{n} \sum\left(\Delta^{2} \ln m_{x}\right)^{2}$ be an indicator of smoothness. The smoothness indicator $\gamma$ can vary from zero to that implied by the raw data. We selected $\gamma=5 \cdot 10^{-6}$ and for each application a corresponding value of the spline parameter $p$ was determined. The selected level of smoothness corresponds approximately to the smoothness of the Kannisto-Makeham model fitted to ages 50 and over.

\section{Results}

The results of mortality forecasting for each population are stored in separate Excel files, named as follows:

\begin{tabular}{|l|l|l|}
\hline Country & Males & Females \\
\hline Belgium & MBEL.XLS & FBEL.XLS \\
\hline Canada & MCAN.XLS & FCAN.XLS \\
\hline England and Wales & MENW.XLS & FENW.XLS \\
\hline France & $\underline{\text { MFRA.XLS }}$ & FFRA.XLS \\
\hline Germany, West & MFRG.XLS & FFRG.XLS \\
\hline Italy & MITA.XLS & FITA.XLS \\
\hline Japan & $\underline{\text { MJPN.XLS }}$ & FJPN.XLS \\
\hline Spain & $\underline{\text { MESPTNP.XLS }}$ & FESPTNP.XLS \\
\hline Switzerland & MCHE.XLS & FCHE.XLS \\
\hline United States & MUSA.XLS & FUSA.XLS \\
\hline
\end{tabular}

Each file includes
a) life tables based both on raw and smoothed death rates,
b) rates of mortality improvement, and
c) raw data on deaths and exposure estimates.

Several diagnostic graphs for each population are provided, too. To start browsing, use the Internet Browser (e.g., MS Internet Explorer) to open index.figures.html.

\footnotetext{
${ }^{7}$ This is a standard divided difference (de Boor 2001) $[x, x+1, x+2] \ln m_{x}$. In our case, computations can be simplified as death rates given by single age.
} 


\section{References}

1. 2003 Technical Panel on Assumptions and Methods. Report to the Social Security Advisory Board. 2003 Oct.

2. Alderson, M. and Ashwood, F. Projection of Mortality Trends for the Elderly. Population Trends. 1985.

3. Alho, Juha M. Effect of Aggregation on the Estimations of Trends in Mortality. Mathematical Population Studies. 1991; 3(1):53-67.

4. Alho, Juha M. Discussion (on Lee, R.: The Lee-Carter Method for Forecasting Mortality, with Various Extensions and Applications). North American Actuarial Journal. 2000; 4:91-93.

5. Bayo, F. R. and McKay, S. F. United States Population Projections for OASDI Cost Estimates. Actuarial Study no. 72. Social Security Administration. Office of the Actuary; 1974 Jul.

6. Bayo, F. R.; Shiman, H. W., and Sobus, B. R. United States Population Projections for OASDHI Cost Estimates. Actuarial Study no. 77. Social Security Administration. Office of the Actuary; 1978 Jun.

7. Bayo, F. R. and Wilkin, J. C. Experience of Disabled-Worker Benefits Under OASDI, 196574. Actuarial Study no. 74. Social Security Administration. Office of the Actuary; 1977 Jan.

8. Bell, Felicitie C. and Wade, Alice H. Life Tables for the United States Social Security Area 1900-2100. Actuarial Study No. 116. U.S. Department of Health and Human Services. Social Security Administration. Office of the Actuary; 2002 Sep 24.

9. Bell, Felicitie C.; Wade, Alice H., and Goss, Stephen C. Life Tables for the United States Social Security Area. Actuarial Study M. 107. U.S. Department of Health and Human Services. Social Security Administration. Office of the Actuary; 1992 Aug.

10. Bell, William R. Comparing and Assessing Time Series Methods for Forecasting AgeSpecific Fertility and Mortality Rates. Journal of Official Statistics. 1997; 13(3):279303.

11. Bongaarts, John. Long Range Trends in Adult Mortality: Models and Projection Methods. Working Paper. Annual Meeting of the Population Association of America, Boston, 2004.

12. Booth, Heather; Maindonald, John, and Smith, Len. Applying Lee-Carter Under Conditions of Variable Mortality Decline. Population Studies. 2002; 56(3).

13. Brass, W. On the Scale of Mortality. Brass, W. Biological Aspects of Demography. London: Taylor \& Francis; 1971.

14. Buettner, Thomas. Approaches and Experiences in Projecting Mortality Patterns for the Oldest Old. North American Actuarial Journal. 2002 Jul; 6(3). 
15. Caselli, Graziella. Future Longevity Among the Elderly. Caselli, Graziella and Lopez, A. D. Health and Mortality among Elderly Populations. Oxford: Clarendon Press; 1996; pp. 235-265.

16. Chariot, P.; Witt, K.; Pautot, V.; Porcher, R.; Thomas, G.; Zafrani, E. S., and Lemaire, F. Declining Autopsy Rate in a French Hospital: Physician's Attitudes to the Autopsy and Use of Autopsy Material in Research Publications. Arch Pathol Lab Med. 2000 May; 124(5):739-45.

17. Coale, Ansley J. and Demeny, Paul with B. Vaughan. Regional Model Life Tables and Stable Population. NewYork: Academic Press; 1983.

18. Coale, Ansley J. and Guang, Guo. Revised Regional Model Life Tables at Very Low Levels of Mortality. Population Index. 1989; 55.

19. Cruijsen, Harri and Eding, Harold. The Latest Mortality Forecasts in the European Union. Tabeau, Ewa; van den Berg Jeths, Anneke, and Heathcote, Christopher eds. Forecasting Mortality in Developed Countries. Kluwer Academic Publishers; 2001.

20. de Boor, Carl. A Practical Guide to Splines. Revised Edition, Applied Mathematical Sciences Vol 27. New York: Springer; 2001; ISBN: 0-387-95366-3.

21. Ewbank, D. C.; Gomez De Leon, J. C., and Stoto, M. A. A Reducible Four-Parameter System of Model Life Tables. Population Studies. 1983; 37:105-127.

22. Hannerz, Harald. An Extension of Relational Methods in Mortality Estimations. Demographic Research. 2001; 4(10).

23. Heligman, L. and Pollard, J. H. The Age Pattern of Mortality. Journal of the Institute of Actuaries. 1980; 107(1):49-80.

24. Himes, Christine L.; Preston, Samuel H., and Condran, Gretchen A. A Relational Model of Mortality at Older Ages in Low Mortality Countries. Population Studies. 1994; 48:269-291.

25. Human Mortality Database. Available online at www.mortality.org: University of California, Berkeley, United States and Max Planck Institute for Demographic Research, Germany; 2004.

26. Keyfitz, Nathan. Choice of Function for Mortality Analysis: Effective Forecasting Depends on a Minimum Parameter Representation. Theoretical Population Biology. 1982; $21: 329-52$.

27. ---. Applied Mathematical Demography. Berlin: Springer-Verlag; 1985; ISBN: 3-540-961550 .

28. Ledermann, S. Nouvelles Tables-Types de Mortalité. Paris: Institut national d'études demographiques; 1969.

29. Lee, Ronald D. The Lee-Carter Method for Forecasting Mortality, with Various Extensions and Applications. North American Actuarial Journal. 2000; 4(1):80-93. 
30. Lee, Ronald D. and Carter, Lawrence R. Modeling and Forecasting U.S. Mortality. Journal of the American Statistical Association. 1992 Sep; 87(419):659-675.

31. Lee, Ronald D. and Miller, Timothy. Evaluating the Performance of the Lee-Carter Method for Forecasting Mortality. Demography. 2001 Nov 4; 38.

32. Lindstrom, P.; Janzon, L., and Sternby, N. H. Declining Autopsy Rate in Sweden: a Study of Causes and Consequences in Malmo, Sweden. J Intern Med. 1997 Aug; 242(2):15765.

33. Lopez, Alan D.; Salomon, Joshua; Ahmad, Omar; Murray, Christopher JL, and Mafat, Doris. Life Tables for 191 Countries: Data, Methods and Results. World Health Organization. Discussion Paper No. 9. 2000.

34. Loughrey, M. B.; McCluggage, W. G., and Toner, P. G. The Declining Autopsy Rate and Clinicians' Attitudes. Ulster Med J. 2000 Nov; 69(2):83-9.

35. Manton, Kenneth G.; Stallard, Eric, and Tolley, H. Dennis. Limits to Human Life Expectancy: Evidence, Prospects, and Implications. Population and Development Review. 1991 Dec; 17(4):603-637; ISSN: 00987921.

36. McNown, R. Modeling and Forecasting U.S. Mortality: Comment. Journal of the American Statistical Association. 1992; 87(419):671-672.

37. McNown, R. and Rogers, A. Forecasting Mortality: A Parameterized Time Series Approach. Demography. 1989 Nov 4; 26(4):645-660.

38. McNown, R. and Rogers, A. Forecasting Cause-Specific Mortality Using Time Series Methods. International Journal of Forecasting. 1992; 8:413-432.

39. Murray, C. J. L.; Ferguson, B. D.; Lopez, A. D.; Guillot, M.; Salomon, J. A., and Ahmad, O. Modified Logit Life Table System: Principles, Empirical Validation, and Application. Population Studies. 2003 Jul; 57(2):165-182.

40. Oeppen, Jim and Vaupel, James W. Broken Limits to Life Expectancy. Science. 2002 May 10; 296.

41. Olshansky, Jay S. On Forecasting Mortality. The Milbank Quarterly. 1988; 3:482-530.

42. Pollard, John H. Projection of Age-Specific Mortality Rates. Population Bulletin of the United Nations 21, No. 22. 1987:55-69.

43. Preston, Samuel H. An Evaluation of Postwar Mortality Projections in Australia, Canada, Japan, New Zealand and the United States. World Health Statistical Report. Special Subject II. Geneva, World Health Organization. 1974.

44. Preston, Samuel H.; Heuveline, Patrick, and Guillot, Michel. Demography: Measuring and Modeling Population Processes. Blackwell Publishers; 2000; ISBN: 1-55786-451-9 (Paperback).

45. Thatcher, Roger A. The Long-Term Pattern of Adult Mortality and the Highest Attained Age. Journal of the Royal Statistical Society: Series A (Statistics in Society). 1999; 
162(1):1.

46. Thatcher, Roger A.; Kannisto, V., and Vaupel, J. W. The Force of Mortality at Ages 80 to 120. Odense, Denmark: Odense University Press; 1998.

47. United Nations. Model Life Tables for Developing Countries. New York: United Nations; 1982.

48. ---. World Population Prospects: The 1998 Revision: Volume III: Analytical Report. New York: United Nations.; 2000a.

49. ---. World Population Prospects: The 2000 Revision: Population Database. Online Database: http://esa.un.org/unpp/. Accessed 19 August 2002. 2000b.

50. ---. World Population Prospects: The 2000 Revision: Volume I: Comprehensive Tables. New York: United Nations.; 2001a.

51. ---. World Population Prospects: The 2000 Revision: Volume III: Analytical Report. New York: United Nations.; 2001b.

52. ---. World Population Prospects: The 2002 Revision: Volume I: Comprehensive Tables. New York: United Nations.; 2003.

53. ---. World Population in 2300. Proceedings of the United Nations Expert Meeting on World Population in 2300. 2004 Mar 24.

54. Van Den Berg Jeths, Anneke; Hoogenveen, Rudolf; De Hollander, Guus, and Tabeau, Ewa. A Review of Epidemiological Approaches to Forecasting Mortality and Morbidity. Tabeau, Ewa; van den Berg Jeths, Anneke, and Heathcote, Christopher eds. Forecasting Mortality in Developed Countries. Kluwer Academic Publishers; 2001.

55. Van Hoorn, Wim and De Beer, Joop. Projecting Mortality in Population Forecasts in the Netherlands. Tabeau, Ewa; van den Berg Jeths, Anneke, and Heathcote, Christopher eds. Forecasting Mortality in Developed Countries. Kluwer Academic Publishers; 2001 .

56. Vaupel, James W. How Change in Age-specific Mortality Affects Life Expectancy. Population Studies. 1986; 40:147-157.

57. Wilmoth, John R. Are Mortality Projections Always More Pessimistic When Disaggregated by Cause of Death? Mathematical Population Studies. 1995; 5(4):293-319.

58. Wilmoth, John R. Is the Pace of Japanese Mortality Decline Converging Toward International Trends? Population and Development Review. 1998; 24(3):593-600.

59. Wolfson, Michael. POHEM: A Framework for Modelling and Understanding the Health of Human Population. World Health Statistics Quarterly. 1994; 47(3/4):157-176.

60. Woodbury, M. A. and Manton, Kenneth G. A Random Walk Model of Human Mortality and Aging. Theoretical Population Biology. 1977; 11:37-48.

61. Yashin, Anatoli I.; Manton, Kenneth G., and Stallard, Eric. Dependent Competing Risks: A 
Stochastic Process Model. Mathematical Biology. 1986; 24:119-40.

62. Zaba, Basia. The Four-Parameter Logit Life-Table System. Population Studies. 1979.

63. ---. Relational Models : Their Uses in Demography. Centre for Population Studies. London School of Hygiene \& Tropical Medicine. CPS Research Paper ; 89-1. 1989. 